\title{
Growth and physiological responses of subalpine forbs to nitrogen and soil moisture: investigating the potential roles of plant functional traits
}

\section{Authors: Anthony H. Slominski, Zac German, and Laura A. Burkle}

The final publication is available at Springer via http://dx.doi.org/10.1007/s11258-018-0848-9.

Slominski, Anthony H. , Zac German, and Laura A. Burkle. "Growth and physiological responses of subalpine forbs to nitrogen and soil moisture: investigating the potential roles of plant functional traits." Plant Ecology 219, no. 8 (June 2018): 941-956. DOI:10.1007/s11258-018-0848-9. 


\title{
Growth and physiological responses of subalpine forbs to nitrogen and soil moisture: investigating the potential roles of plant functional traits
}

\author{
Anthony H. Slominski ${ }^{\circledR}$ - Zac German • Laura A. Burkle
}

\begin{abstract}
Anthropogenic inputs of biologically available nitrogen $(\mathrm{N})$ and climate change are simultaneously altering $\mathrm{N}$ and soil moisture availability in terrestrial ecosystems. Yet, plant responses to concurrent changes in both $\mathrm{N}$ and soil moisture in non-grassland ecosystems remain poorly understood. Our objective was to investigate how rooting depth and $\mathrm{N}$-fixing ability - two functional traits we expected to mediate soil moisture and $\mathrm{N}$ limitations - influence forb responses to $\mathrm{N}$ and soil moisture availability in the Rocky Mountains USA. We assessed the growth and physiological responses (i.e., chlorophyll fluorescence, transpiration rate, and floral display) of four subalpine forb species to $\mathrm{N}$ additions across a naturally-occurring soil moisture gradient during one growing season. Soil moisture had a stronger positive effect on growth in shallow-rooted species and $\mathrm{N}$ additions had a stronger positive effect on photosynthetic capacity in species without $\mathrm{N}$-fixing abilities. Transpiration rates were not consistent with soil moisture limitations expected for shallow-rooted species, and soil moisture and $\mathrm{N}$ had a neutral or negative influence on maximum floral displays across species. Nitrogen and soil moisture appeared to each limit separate response variables in some cases and we did not observe any $\mathrm{N} 9$ soil moisture interactions. These findings suggest that shallow-rooted species may be more vulnerable to increased drought severity and that increased $\mathrm{N}$ availability may disproportion-ately benefit species without $\mathrm{N}$ fixing abilities. However, mixed support for our hypotheses suggests that environmental conditions and functional traits not evaluated here likely influence subalpine plant responses to soil moisture and $\mathrm{N}$ availability.
\end{abstract}




\section{Introduction}

In terrestrial ecosystems, biologically available nitrogen (N) is increasing (e.g., Fowler et al. 2013). Simultaneously, climate change is affecting soil moisture availability by altering precipitation patterns and snowpack dynamics (Dai 2013; Stewart 2009; IPCC 2014). The concentrations of these key limiting resources can strongly influence plant abundances and plant community composition (e.g., Knapp et al. 2002; Bobbink et al. 2010; Smith et al. 2015). Thus, understanding plant responses to changes in $\mathrm{N}$ and soil moisture availability is a central goal in plant ecology (Vitousek 1997; Chapin et al. 2000; Hooper et al. 2005; Pecl et al. 2017).

Plant responses to changing $\mathrm{N}$ and soil moisture concentrations will likely be determined by competition for limiting resources, which will be mediated by resource limitations that reflect tradeoffs in functional traits (Lavorel and Garnier 2002; Grime 2006; Dybzinski and Tilman 2007). For example, shallowrooted plant species may be poor competitors for soil moisture, but may allocate resources to other functions, conferring an advantage when soil moisture is adequate (Ho et al. 2005). In addition, species with $\mathrm{N}$-fixing abilities may have a competitive advantage in $\mathrm{N}$-limited systems (Shantz et al. 2016), but may lose this competitive edge and decline in abundance as $\mathrm{N}$ availability increases (Suding et al. 2005; Skogen et al. 2011). Investigating the influence of functional traits on outcomes in competition for $\mathrm{N}$ and soil moisture can inform our understanding of how plant abundances and community composition will respond to changing concentrations of these limiting resources (Zavaleta et al. 2003; Parmesan and Hanley 2015; Wei et al. 2017).

The effects of anthropogenic $\mathrm{N}$ deposition and altered soil moisture availability on plant communities may be particularly pronounced in temperate montane systems (Harte and Shaw 1995), where increasing N levels (Williams et al. 1996; Bowman et al. 2006) and increased summer drought severity are expected (Harte et al. 1995; Mote 2006; Inouye 2008). However, the majority of studies investigating plant responses to changing $\mathrm{N}$ and soil moisture have focused on grassland systems (e.g., Foster and Gross 1998; Knapp et al. 2002; Stevens et al. 2004; Clark and Tilman 2008; Dickson et al. 2014, Hoover et al. 2017). Moreover, most of these studies have isolated the effects of either $\mathrm{N}$ or soil moisture. Yet, plant responses to simultaneous changes in multiple resources can be complex (e.g., De Valpine and Harte 2001; Dukes et al. 2005; Harpole et al. 2007; Reich et al. 2014; Vourlitis 2017; Yue et al. 2017) and conclusions about plant responses based on singleresource manipulations may be misleading (Sala et al. 2000; Cleland and Harpole 2010).

Here, we conducted field manipulations of $\mathrm{N}$ across a natural soil moisture gradient in the Rocky Mountains, USA during one growing season in 2009 and recorded growth and physiological responses (i.e., photosynthetic capacity, transpiration rate, and maximum floral display) of four subalpine forb species. We assessed growth and physiological responses to evaluate multiple mechanisms by which forbs may respond to different levels of $\mathrm{N}$ and soil moisture. Focal forb species had different rooting depths (i.e., short fibrous roots vs. deeper tap-roots) and represented either $\mathrm{N}$-fixing legume species or non-legume species lacking $\mathrm{N}$-fixing abilities (i.e., four species, one of each combination of phenotypes). Our objective was to evaluate the influence of rooting depth and $\mathrm{N}$-fixing ability - two functional traits we expected to mediate soil moisture and $\mathrm{N}$ limitations - on subalpine forb responses to $\mathrm{N}$ and soil moisture availability. Our design allowed a comparison of how short-term growth and physiological responses to $\mathrm{N}$ are mediated by long-term exposure to varying soil moisture concentrations among forb species with contrasting functional traits.

We expected shallow-rooted species to be more water-limited compared to deeper-rooted species (Fitter et al. 1991; Lynch 1995). Thus, we predicted increased growth, transpiration rates, and floral displays for shallow-rooted species as soil moisture increased. For deeper-rooted species, we expected that growth, transpiration rates, and floral displays would not respond, or decrease as soil moisture increased due to reduced competitive ability when soil moisture is abundant (Gardner 1960; Ho et al. 2005; Jacobs et al. 2009). We expected greater $\mathrm{N}$ limitation in nonlegume forbs compared to legumes. For non-legumes, we predicted $\mathrm{N}$ additions would increase growth, photosynthetic capacity, and floral display (Burkle \& Irwin 2009, 2010), while we expected legumes not to respond to $\mathrm{N}$ additions, or to respond negatively as a result of decreased competitive ability (Suding et al. 2005; Skogen et al. 2011). Finally, we expected an 
interaction between $\mathrm{N}$ and soil moisture in shallowrooted species lacking $\mathrm{N}$-fixing abilities, such that enhanced growth and physiological function would be greatest in individuals receiving $\mathrm{N}$ under high soil moisture conditions (Harpole et al. 2007). Evaluating how functional traits influence growth and physiological responses to soil moisture and $\mathrm{N}$ availability allowed us to speculate about which plant functional groups may experience negative consequences from anthropogenic $\mathrm{N}$ deposition and increased drought severity, and which species may benefit.

\section{Methods}

Focal forb species

We selected four forb species for this study: $\mathrm{He}$ liomeris multiflora (showy goldeneye), Erigeron speciosus (showy fleabane), Lathyrus leucanthus (Rocky Mountain sweetpea), and Lupinus spp. (lupine). Lupinus individuals were not identified to species and were grouped at the genus level because hybridization results in individuals that lack diagnostic characteristics (Cronquist et al. 1972). Lupinus hybrids likely represented two or three species of Lupinus with similar functional traits. We selected these four forb species because they are native to Colorado, are all commonly found in subalpine habitats, have either short fibrous roots or deeper tap-roots, and represent either $\mathrm{N}$-fixing species or species that do not fix N. Heliomeris multiflora and Lupinus spp. have deeper rooting depths compared to E. speciosus and L. leucanthus, and L. leucanthus and Lupinus spp. are legumes with $\mathrm{N}$-fixing abilities, while H. multiflora and E. speciosus lack N-fixing abilities.

Study site

This study was conducted in 2009 between 16 June and 3 August in a relatively treeless subalpine meadow (hereafter Bellview Bench) $\left(39^{\circ} 00^{\prime} 20.58^{\prime \prime} \mathrm{N}\right.$, $107^{\circ} 01^{\prime} 50.70^{\prime \prime} \mathrm{W}$ ) at 3150 meters of elevation, approximately $6.5 \mathrm{~km}$ north-west of the Rocky Mountain Biological Lab (RMBL) in Gothic, CO USA. A low rate of $\mathrm{N}$ deposition near the RMBL (ca. $2 \mathrm{~kg} \mathrm{~N} \mathrm{ha}^{-1}$ year $^{-1}$; CASTNET 2013) provided an opportunity to explore the effects of $\mathrm{N}$ additions on plants likely experiencing $\mathrm{N}$ limitation. Bellview
Bench has a naturally-occurring soil moisture gradient with wetter soils near a seasonal stream, and decreasing soil moisture further from the stream and above a large rock outcrop. The aspect at Bellview Bench is south-east with a slope of ca. $15-20^{\circ}$.

\section{Experimental design}

On 16 June, 2009, we used a Campbell Scientific CS658 HydroSense II digital water content sensor with dual $20 \mathrm{~cm}$ sensor rods to measure volumetric soil water content at Bellview Bench. By haphazardly placing the probe at 160 locations throughout the meadow, we identified a soil moisture gradient ranging between 7 and 45\% water. This initial soil moisture survey was conducted during the morning several weeks after snowmelt and following several days without precipitation. While measuring soil moisture at $20 \mathrm{~cm}$ may not extend to the rooting depths of all plant individuals in this study (e.g., some Lupinus argenteus may extend roots deeper than $20 \mathrm{~cm}$; USDA Plant Database, http://www.plants. usda.gov/), previous work showed that differences in soil moisture up to $25 \mathrm{~cm}$ were generally relative to soil moisture near the surface (Harte et al. 1995). Thus, we assume that the soil moisture gradient we measured at Bellview Bench was maintained past $20 \mathrm{~cm}$ and that individuals with roots deeper than $20 \mathrm{~cm}$ were experiencing a soil moisture gradient relative to our measurements.

Forty plants of each species were haphazardly selected across the soil moisture gradient at Bellview Bench for growth and physiological function measurements (see Data Collection below), totaling 160 individual plants. Ten plants of each species were randomly assigned to either $\mathrm{N}$-addition treatments or control treatments receiving no additional $\mathrm{N}$. We measured soil moisture at each plant two times during the study, 19 June and 15 July. We estimated soil moisture availability at each plant over the course of the growing season by taking the mean of these measurements. There was no statistical difference in soil moisture availability between $\mathrm{N}$-addition plants and control plants (Online Resource 1).

$\mathrm{N}$-additions were applied every 7-10 days for 4 weeks (i.e., four additions) starting 28 June by applying ammonium nitrate $\left(\mathrm{NH}_{4} \mathrm{NO}_{3}\right)$ mixed with $250 \mathrm{~mL}$ of water to a $0.5 \times 0.5 \mathrm{~m}$ area surrounding each $\mathrm{N}$-addition plant. We added $2.25 \mathrm{~g} \mathrm{~N} \mathrm{~m}^{-2}$ 
during each $\mathrm{N}$ addition for a total of $9 \mathrm{~g} \mathrm{~N} \mathrm{~m}^{-2}$ over the duration of the study. Applying this amount of $\mathrm{N}$ ensured that our treatments represented a high level of $\mathrm{N}$ input (Bowman et al. 2006), and is comparable to concentrations of $\mathrm{N}$ added by other studies in this system (Burkle \& Irwin 2009; De Valpine \& Harte 2001). This level of supplemental $\mathrm{N}$ likely eliminated $\mathrm{N}$ limitation at each $\mathrm{N}$-addition plant. Plants at Bellview Bench were naturally spaced such that treatments were assumed not to affect neighboring plants and no additional effort was made (e.g., removing or clipping neighboring plants) to prevent treatment effects on neighboring plants. We did not add $250 \mathrm{~mL}$ of water to the control plants during $\mathrm{N}$ additions because this amount is equivalent to $1 \mathrm{~mm}$ of precipitation and was considered negligible. Low ambient humidity and frequent rainfall events totaling ca. $76 \mathrm{~mm}$ of precipitation between 1 June and 3 August during 2009 (NOAA, https://www.ncdc.noaa. gov/) indicate that $\mathrm{N}$ treatments had little to no effect on soil moisture availability.

\section{Data collection}

We recorded plant growth and measures of physiological function in response to $\mathrm{N}$ additions across a soil moisture gradient and viewed positive responses to $\mathrm{N}$ and soil moisture as indicative of release from resource limitation.

Growth was measured by quantifying plant size for each individual four times over the course of the 2009 growing season. Measurements used to calculate plant size were conducted seven to 10 days following each $\mathrm{N}$ treatment between 28 June and 3 August. For $H$. multiflora, E. speciosus, and Lupinus spp., plant size was calculated by multiplying the number of stems by the average stem height in $\mathrm{cm}$ (measured from the ground to the tip) for each individual. Lathyrus leucanthus individuals always have a single stem, and plant size was calculated by multiplying the number of leaves by stem height. For the species with multiple stems, average stem height was calculated by averaging three stem measurements representing a short, medium, and tall stem for that individual. To control for differences in initial plant sizes among individual plants of the same species, percent growth was calculated on each measurement date by dividing plant size by the size calculated for that individual on 28 June (i.e., prior to the start of $\mathrm{N}$ treatments).
Chlorophyll fluorescence is significantly correlated with total energy yield from $\mathrm{CO}_{2}$ fixation during photosynthesis (Leverenz and Öquist 1987; Genty et al. 1989) and has become a standard method for assessing photosynthetic capacity in plant leaves (Baker 2008). Given that N can limit photosynthetic rates, photosynthetic capacity may reflect $\mathrm{N}$ availability and species-specific N limitations (Ball et al. 1995; Van der tol et al. 2014). Chlorophyll fluorescence was measured on 2 July and again on 24 July with a OptiScience OS1-FL pulse modulated chlorophyll fluorometer that quantified the ratio of variable fluorescence to maximal fluorescence $\left(\mathrm{F}_{\mathrm{v}} / \mathrm{F}_{\mathrm{m}}\right)$ in units of $\mu$ mol photons $\mathrm{m}^{-2} \mathrm{~s}^{-1}$. Decreasing ratios of $\mathrm{F}_{\mathrm{v}} / \mathrm{F}_{\mathrm{m}}$ indicate reduced photosynthetic capacity.

Transpiration rate is the measure of water vapor loss through open stomata of leaves (Farquhar and Sharkey 1982) and may indicate leaf water concentrations as leaves adjust stomatal openings to regulate water loss (Chaves et al. 2002; Obidiegwu et al. 2015). Transpiration rates can reflect soil moisture availability (Granier et al. 2000; Tuzet et al. 2003) and speciesspecific soil moisture limitations (Bond and Kavanagh 1999; Sack and Holbrook 2006). Transpiration rates were measured on 8 July and again on 24 July using a Decagon Devices SC-1 leaf porometer that recorded the water vapor flux out of stomata in $\mathrm{mmol} \mathrm{m} \mathrm{m}^{-2} \mathrm{~s}^{-1}$.

Maximum floral display was quantified by counting open flowers on each individual plant on three dates in July, each separated by 7 days. The largest number of open flowers out of the three measurements was used as maximum floral display. Given that total flower production correlates with maximum floral display in subalpine forbs (Inouye et al. 2002), this method reasonably estimates overall flower production of each plant in our study.

\section{Statistical analyses}

All analyses were conducted using $\mathrm{R}$ statistical software version 3.4.3 ( $\mathrm{R}$ core team 2017) and following Zuur et al. (2009). Growth and physiological responses were analyzed individually for each species by fitting separate models to percent growth, chlorophyll fluorescence, transpiration rate, and maximum floral display as individual response variables. A covariance matrix of all response variables suggested that our response variables were not collinear and that fitting separate models to each response 
variable was appropriate (Online Resource 2). Linear mixed effects models were fitted to growth, chlorophyll fluorescence, and transpiration rate using the 'Ime' function in the 'nlme' package (Pinheiro et al. 2013; R core team 2017). This approach accounts for a violation of independence due to repeated measures on the same plant individuals and heterogeneity in the variances of the residuals. To account for overdispersion in floral display counts for E. speciosus, $H$. multiflora, and Lupinus spp., we fit negative binomial generalized linear models with a log link using the 'glm.nb' function in the 'MASS' package (Venables and Ripley 2002). Floral display counts for $L$. leucanthus were Poisson-distributed without overdispersion and were analyzed by fitting a Poissondistributed generalized linear model with a log link using the 'glm' function (R core team 2017).

For all models, $\mathrm{N}$ treatment ( $\mathrm{N}$-addition vs. control) was included as a categorical explanatory variable and mean soil moisture was included as a continuous explanatory variable. Models fitted to percent growth included measurement date as a continuous explanatory variable. Models fitted to chlorophyll fluorescence and transpiration rate included measurement date as a categorical explanatory variable with two levels (i.e., two measurement dates), except for $H$. multiflora transpiration rate, which was only analyzed for 8 July due to data loss for 24 July. Measurement time of day or the ambient air temperature at each measurement was included as covariates in all chlorophyll fluorescence and transpiration models, respectively. Models fitted to maximum floral display included only soil moisture and $\mathrm{N}$ treatment, with plant size as a covariate. All models included all possible two- and three-way interactions among soil moisture, $\mathrm{N}$ treatment, and measurement date. We included two-way interactions between soil moisture and $\mathrm{N}$ treatment to assess evidence of co-limitation by soil moisture and $\mathrm{N}$. Two-way interactions with measurement date were included to assess whether the effect of soil moisture or $\mathrm{N}$ treatment depended on date. Three-way interactions of soil moisture, $\mathrm{N}$, and measurement date were included to assess whether responses to soil moisture across measurement dates differed by $\mathrm{N}$ treatment. All models, excluding models fitted to floral display, included plant individual as a random intercept to account for repeated measures on the same plant individuals. Models fitted to $H$. multiflora and Lupinus spp. percent growth included random slopes for plant individuals across time. When determined to be appropriate following visual inspection of the residuals, models included the 'varIdent' variance structure for $\mathrm{N}$ treatment (i.e., categorical variable) and the 'varFixed' variance structure for each continuous variable (i.e., mean moisture, measurement date, time, temperature, and plant size). The 'varIdent' structure allows $\mathrm{N}$-addition plants and control plants to have different variances and the 'varFixed' structure ensures a variance that is proportional to the values of a continuous explanatory variable when the spread of the residuals increases with the values of the explanatory variable. To account for potential temporal autocorrelation in growth measurements over the growing season, we included the corCAR1 correlation structure, which accounts for a violation of independence among growth measurements occurring in proximity through time.

To determine the relative influence of soil moisture, $\mathrm{N}$ treatment, and their interactions on each response variable, we interpreted significance levels from each full model and reported these results in ANOVA tables. Effect sizes reported in the Results section were obtained by post hoc analyses using the lsmeans function in the lsmeans package (Lenth and Hervac 2015). All figures were produced using the ggplot function in the ggplot2 package (Wickham 2009).

\section{Results}

Erigeron speciosus (shallow roots; non $\mathrm{N}$-fixing)

Soil moisture influenced E. speciosus percent growth (Table 1; Fig. 1a), with percent growth increasing by $1.3 \%$ per each $1 \%$ increase in soil moisture concentration (95\% CI 0.3-2.3\%; Table 2). Marginal evidence suggested that $\mathrm{N}$ treatment influenced $E$. speciosus percent growth (Table 1; Fig. 1a), with $\mathrm{N}$ additions increasing growth by $14.0 \%$ compared to control plants $\left(t_{1,33}=1.84, p=0.074 ;\right.$ Table 2$)$. There were no significant interactions among soil moisture, $\mathrm{N}$ treatment, and measurement date on $E$. speciosus percent growth (Table 1).

After accounting for measurement time, we observed a marginally significant interaction between soil moisture and measurement date on E. speciosus chlorophyll fluorescence (Table 1; Fig. 1b). On 2 July, $\mathrm{F}_{\mathrm{v}} / \mathrm{F}_{\mathrm{m}}$ increased by $0.001 \mu \mathrm{mol}$ photons $\mathrm{m}^{-2} \mathrm{~s}^{-1}$ per 
Table 1 ANOVA tables of the linear mixed effects models and generalized linear models of each response variable by species

\begin{tabular}{|c|c|c|c|c|c|c|c|c|c|c|c|c|c|}
\hline & \multicolumn{3}{|c|}{$\begin{array}{l}\text { percent } \\
\text { growth }\end{array}$} & \multicolumn{3}{|c|}{$\begin{array}{l}\text { chlorophyll } \\
\text { fluorescence }\end{array}$} & \multicolumn{3}{|c|}{$\begin{array}{l}\text { transpiration } \\
\text { rate }\end{array}$} & \multicolumn{4}{|c|}{$\begin{array}{l}\text { floral } \\
\text { display }\end{array}$} \\
\hline & $d f$ & $F$ & $P$ & $d f$ & $F$ & $P$ & $d f$ & $F$ & $P$ & Res.dj & $f D e v$. & Res.Dev & $P$ \\
\hline \multicolumn{14}{|l|}{ E. speciosus } \\
\hline measurement date & 1,106 & 95.93 & $<0.001$ & 1,26 & 171.6 & $<0.001$ & 1,16 & 63.43 & $<0.001$ & - & - & - & - \\
\hline soil moisture & 1,33 & 7.18 & 0.011 & 1,33 & 1.33 & 0.257 & 1,32 & 0.49 & 0.488 & 1,35 & 4.27 & 56.18 & 0.039 \\
\hline $\mathrm{N}$ treatment & 1,33 & 3.42 & 0.074 & 1,33 & 18.1 & $<0.001$ & 1,32 & 0.83 & 0.370 & 1,34 & 1.93 & 54.25 & 0.165 \\
\hline plant size & - & - & - & - & - & - & - & - & - & 1,33 & 6.09 & 48.17 & 0.014 \\
\hline measurement time & - & - & - & 1,26 & 0.47 & 0.498 & - & - & - & - & - & - & - \\
\hline temperature & - & - & - & - & - & - & 1,16 & 8.22 & 0.011 & - & - & - & - \\
\hline date $\mathrm{x} N$ & 1,106 & 0.00 & 0.952 & 1,26 & 0.01 & 0.943 & 1,16 & 3.00 & 0.102 & - & - & - & - \\
\hline date $\mathrm{x}$ soil moisture & 1,106 & 0.31 & 0.581 & 1,26 & 4.19 & 0.051 & 1,16 & 4.51 & 0.050 & - & - & - & - \\
\hline soil moisture $\mathrm{x} N$ & 1,109 & 0.07 & 0.794 & 1,33 & 0.11 & 0.748 & 1,32 & 0.16 & 0.697 & 1,32 & 2.77 & 45.40 & 0.096 \\
\hline date $\mathrm{x}$ moisture $\mathrm{x} \mathrm{N}$ & 1,106 & 1.35 & 0.247 & 1,26 & 0.31 & 0.585 & 1,16 & 1.06 & 0.319 & - & - & - & - \\
\hline \multicolumn{14}{|l|}{ H. multiflora } \\
\hline measurement date* & 1,101 & 61.22 & $<0.001$ & 1,20 & 6.29 & 0.021 & $\mathrm{NA}^{*}$ & NA* & $\mathrm{NA}^{*}$ & - & - & - & - \\
\hline soil moisture & 1,32 & 1.20 & 0.281 & 1,33 & 1.46 & 0.235 & 1,33 & 0.51 & 0.479 & 1,33 & 8.02 & 50.49 & $<0.001$ \\
\hline $\mathrm{N}$ treatment & 1,32 & 0.07 & 0.792 & 1,33 & 3.68 & 0.064 & 1,33 & 0.10 & 0.751 & 1,32 & 0.48 & 50.01 & 0.489 \\
\hline plant size & - & - & - & - & - & - & - & - & - & 1,31 & 4.43 & 45.58 & 0.035 \\
\hline measurement time & - & - & - & 1,20 & 2.99 & 0.099 & - & - & - & - & - & - & - \\
\hline temperature & - & - & - & - & - & - & 1,33 & 5.64 & 0.024 & - & - & - & - \\
\hline date $\mathrm{x} N$ & 1,101 & 0.09 & 0.765 & 1,20 & 0.00 & 0.947 & $\mathrm{NA}^{*}$ & NA* & $\mathrm{NA}^{*}$ & - & - & - & - \\
\hline date $\mathrm{x}$ soil moisture & 1,101 & 2.21 & 0.140 & 1,20 & 2.21 & 0.152 & $\mathrm{NA}^{*}$ & NA* & $\mathrm{NA}^{*}$ & - & - & - & - \\
\hline soil moisture $\mathrm{x} N$ & 1,32 & 0.94 & 0.340 & 1,33 & 1.22 & 0.278 & 1,33 & 0.06 & 0.807 & 1,30 & 4.55 & 41.03 & 0.033 \\
\hline date $\mathrm{x}$ moisture $\mathrm{x} N$ & 1,101 & 0.24 & 0.626 & 1,20 & 0.11 & 0.740 & NA* & NA* & NA* & - & - & - & - \\
\hline \multicolumn{14}{|l|}{ L. leucanthus } \\
\hline measurement date & 1,42 & 0.67 & 0.418 & 1,10 & 42.41 & $<0.001$ & 1,19 & 7.52 & 0.013 & - & - & - & - \\
\hline soil moisture & 1,19 & 3.83 & 0.065 & 1,31 & 0.02 & 0.887 & 1,29 & 0.04 & 0.844 & 1,25 & 9.97 & 34.84 & 0.002 \\
\hline $\mathrm{N}$ treatment & 1,19 & 1.32 & 0.265 & 1,31 & 8.07 & 0.008 & 1,29 & 0.21 & 0.651 & 1,24 & 2.87 & 31.96 & 0.090 \\
\hline plant size & - & - & - & - & - & - & - & - & - & 1,23 & 11.79 & 20.17 & $<0.001$ \\
\hline measurement time & - & - & - & 1,10 & 3.04 & 0.112 & - & - & - & - & - & - & - \\
\hline temperature & - & - & - & - & - & - & 1,19 & 0.03 & 0.855 & - & - & - & - \\
\hline date $\times \mathrm{N}$ & 1,42 & 0.64 & 0.430 & 1,10 & 8.52 & 0.015 & 1,19 & 0.08 & 0.782 & - & - & - & - \\
\hline date $\mathrm{x}$ soil moisture & 1,42 & 0.10 & 0.757 & 1,10 & 1.08 & 0.324 & 1,19 & 0.63 & 0.436 & - & - & - & - \\
\hline $\mathrm{N} x$ soil moisture & 1,19 & 1.76 & 0.200 & 1,31 & 0.01 & 0.919 & 1,29 & 0.44 & 0.510 & 1,22 & 0.10 & 20.07 & 0.751 \\
\hline date $\mathrm{x}$ moisture $\mathrm{x} \mathrm{N}$ & 1,42 & 0.24 & 0.627 & 1,10 & 0.15 & 0.710 & 1,19 & 0.54 & 0.472 & - & - & - & - \\
\hline \multicolumn{14}{|l|}{ Lupinus spp. } \\
\hline measurement date & 1,101 & 94.18 & $<0.001$ & 1,26 & 33.37 & $<0.001$ & 1,26 & 48.93 & $<0.001$ & - & - & - & - \\
\hline soil moisture & 1,31 & 1.48 & 0.232 & 1,32 & 2.51 & 0.123 & 1,32 & 2.38 & 0.133 & 1,34 & 3.81 & 80.96 & 0.051 \\
\hline $\mathrm{N}$ treatment & 1,31 & 0.52 & 0.477 & 1,32 & 0.10 & 0.754 & 1,32 & 0.05 & 0.822 & 1,33 & 2.67 & 78.29 & 0.102 \\
\hline plant size & - & - & - & - & - & - & - & - & - & 1,32 & 39.18 & 39.11 & $<0.001$ \\
\hline measurement time & - & - & - & 1,26 & 0.56 & 0.459 & - & - & - & - & - & - & - \\
\hline temperature & - & - & - & - & - & - & 1,26 & 9.73 & 0.004 & - & - & - & - \\
\hline date $\mathrm{x} N$ & 1,101 & 0.66 & 0.420 & 1,26 & 0.14 & 0.715 & 1,26 & 1.42 & 0.244 & - & - & - & - \\
\hline date $\mathrm{x}$ soil moisture & 1,101 & 0.00 & 0.955 & 1,26 & 0.38 & 0.544 & 1,26 & 0.28 & 0.603 & - & - & - & - \\
\hline soil moisture $\mathrm{x} N$ & 1,31 & 0.32 & 0.578 & 1,32 & 0.78 & 0.384 & 1,32 & 0.97 & 0.331 & 1,31 & 0.66 & 38.45 & 0.418 \\
\hline date $\mathrm{x}$ moisture $\mathrm{x} \mathrm{N}$ & 1,101 & 0.12 & 0.733 & 1,26 & 1.45 & 0.239 & 1,26 & 1.09 & 0.306 & - & - & - & - \\
\hline
\end{tabular}

$P$-values significant at $\alpha<0.050$ are shown in bold

Dashes indicate explanatory variables that were not included in that model

NA* indicates $H$. multiflora transpiration data lost for 24 July 

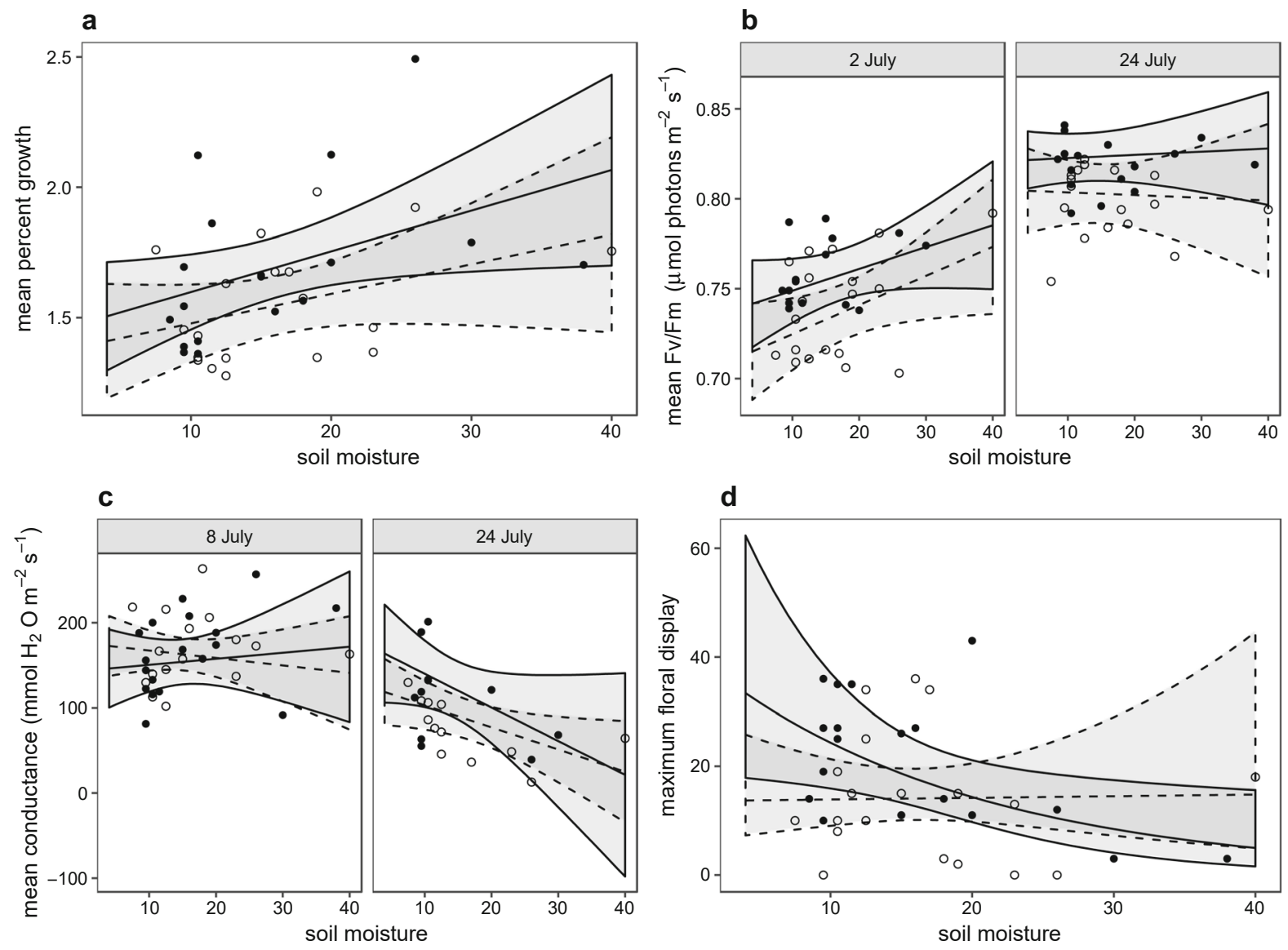

Fig. 1 Erigeron speciosus. Least squares means and 95\% confidence bands for $\mathrm{N}$-addition (solid lines) and control plants (dashed lines) across soil moisture for a percent growth, $\mathbf{b}$ chlorophyll fluorescence, $\mathbf{c}$ transpiration rate, and $\mathbf{d}$ maximum

each $1 \%$ increase in soil moisture concentration $(95 \%$ CI $0.0003-0.0025 \mu \mathrm{mol}$ photons $\mathrm{m}^{-2} \mathrm{~s}^{-1}$ increase; Table 2), while the change in $F_{v} / F_{m}$ across soil moisture on 24 July was not different from zero (95\% CI -0.0009 to $0.0009 \mu \mathrm{mol}$ photons $\mathrm{m}^{-2} \mathrm{~s}^{-1}$; Table 2). N treatment influenced $E$. speciosus chlorophyll fluorescence (Table 1; Fig. 1b), with $\mathrm{N}$ additions increasing $\mathrm{F}_{\mathrm{v}} / \mathrm{F}_{\mathrm{m}}$ by $0.021 \mu \mathrm{mol}$ photons $\mathrm{m}^{-2} \mathrm{~s}^{-1}$ compared to control plants $\left(t_{1,27}=4.34, p<0.001\right.$; Table 2). There was no significant interaction between soil moisture and $\mathrm{N}$ treatment or $\mathrm{N}$ treatment and measurement date, and no significant three-way interaction of soil moisture, $\mathrm{N}$ treatment, and measurement date on E. speciosus chlorophyll fluorescence (Table 1).

floral display. Observed data for $\mathrm{N}$-addition (filled circles) and control plants (open circles) are averaged across measurement dates in growth figures

After accounting for temperature, we observed a marginally significant interaction between soil moisture and measurement date on E. speciosus transpiration rate (Table 1; Fig. 1c). On 24 July, transpiration rate decreased by $3.279 \mathrm{mmol} \mathrm{H}_{2} \mathrm{O} \mathrm{m}^{-2} \mathrm{~s}^{-1}$ per each $1 \%$ increase in soil moisture concentration $(95 \% \mathrm{CI}$

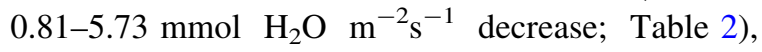
while the change in transpiration rate across soil moisture on 8 July was not different from zero (95\% $\mathrm{CI}-2.307$ to $2.138 \mathrm{mmol}_{2} \mathrm{O} \mathrm{m}^{-2} \mathrm{~s}^{-1}$; Table 2). There was no effect of $\mathrm{N}$ treatment, no significant interaction between soil moisture and $\mathrm{N}$ treatment or $\mathrm{N}$ treatment and measurement date, and no significant three-way interaction of soil moisture, $\mathrm{N}$ treatment, and measurement date on E. speciosus transpiration rate (Table 1). 
Table 2 Effect size coefficients generated by post hoc analyses of each response variable across species

\begin{tabular}{|c|c|c|c|c|c|c|c|c|c|c|c|c|c|c|c|c|}
\hline & $\begin{array}{l}\text { Percer } \\
\text { growt }\end{array}$ & & & & $\begin{array}{l}\text { Chlor } \\
\text { fluore }\end{array}$ & $\begin{array}{l}\text { ophyll } \\
\text { scence }\end{array}$ & & & $\begin{array}{l}\text { Transp } \\
\text { rate }\end{array}$ & iratio & & & $\begin{array}{l}\text { Floral } \\
\text { display }\end{array}$ & & & \\
\hline & $\begin{array}{l}\text { E. } \\
\text { spec. }\end{array}$ & $\begin{array}{l}H . \\
\text { mult. }\end{array}$ & $\begin{array}{l}L . \\
\text { leuc. }\end{array}$ & $\begin{array}{l}\text { Lup. } \\
\text { spp. }\end{array}$ & $\begin{array}{l}\text { E. } \\
\text { spec. }\end{array}$ & $\begin{array}{l}H . \\
\text { mult. }\end{array}$ & $\begin{array}{l}L . \\
\text { leuc. }\end{array}$ & $\begin{array}{l}\text { Lup. } \\
\text { spp. }\end{array}$ & $\begin{array}{l}\text { E. } \\
\text { spec. }\end{array}$ & $\begin{array}{l}\text { H. } \\
\text { mult. }\end{array}$ & $\begin{array}{l}L . \\
\text { leuc. }\end{array}$ & $\begin{array}{l}\text { Lup. } \\
\text { spp. }\end{array}$ & $\begin{array}{l}\text { E. } \\
\text { spec. }\end{array}$ & $\begin{array}{l}H . \\
\text { mult. }\end{array}$ & $\begin{array}{l}L . \\
\text { leuc. }\end{array}$ & $\begin{array}{l}\text { Lup. } \\
\text { spp. }\end{array}$ \\
\hline soil moisture & $\begin{array}{l}0.013 \\
* *\end{array}$ & - & - & - & - & - & - & - & - & - & - & - & - & - & $\begin{array}{l}-0.140 \\
* *\end{array}$ & $\begin{array}{l}-0.019 \\
*\end{array}$ \\
\hline $\mathrm{N}$ treatment & $\begin{array}{l}0.140 \\
*\end{array}$ & - & - & - & $\begin{array}{l}0.021 \\
* * *\end{array}$ & $\begin{array}{l}0.014 \\
*\end{array}$ & - & - & - & - & - & - & - & - & - & - \\
\hline moisture $\mathrm{x} \mathrm{N}$ & - & - & - & - & - & - & - & - & - & - & - & - & $\begin{array}{l}-0.056 \\
*\end{array}$ & $\begin{array}{l}0.081 \\
* *\end{array}$ & - & - \\
\hline moisture $\mathrm{x}$ date & - & - & - & - & $\begin{array}{l}0.001 \\
* *\end{array}$ & - & - & - & $\begin{array}{l}-3.190 \\
* *\end{array}$ & NA & - & - & NA & NA & NA & NA \\
\hline $\mathrm{N} x$ date & - & - & - & - & - & - & $\begin{array}{l}0.036 \\
* * *\end{array}$ & - & - & NA & - & - & NA & NA & NA & NA \\
\hline
\end{tabular}

Coefficients indicate the following effects: "soil moisture"- the change per each $1 \%$ increase in soil moisture concentration (i.e., moisture slope); "N treatment" $-\mathrm{N}$-addition minus control plants; "moisture $\times \mathrm{N}$ "-moisture slope for $\mathrm{N}$-addition minus moisture slope for control plants; "moisture $\times$ date" - the moisture slope on the earlier measurement date minus the moisture slope on the later measurement date; " $\mathrm{N} \times$ date" - the difference between $\mathrm{N}$-addition minus control plants on the earlier measurement date compared to the later measurement date

Dash marks represent effects not significant at the 0.100 level and NA denote parameters not included in that model. Shaded cells corresponding to soil moisture, $\mathrm{N}$, and soil moisture $\times \mathrm{N}$ interactions indicate responses that support the stated hypothesis for that species and response. Open cells indicate responses that oppose the stated hypothesis, except for interactions with date, where a priori hypotheses were not made

Asterisks denote the significance of each effect size

*Significance at $p=0.050-0.0100$

$* *$ Significance at $p=0.001-0.049$

$* * *$ Significance at $p<0.001$

After accounting for plant size, we observed a marginally significant interaction between soil moisture and $\mathrm{N}$ treatment on E. speciosus maximum floral display (Table 1; Fig. 1d). $\mathrm{N}$ additions decreased floral display by $5.3 \%$ per each $1 \%$ increase in soil moisture (95\% CI $0.8-10.0 \%$ decrease; Table 2), while the change in floral display across soil moisture for control plants was not different from zero $(95 \% \mathrm{CI}$ -4.2 to $4.6 \%$; Table 2).

\section{Heliomeris multiflora (deeper roots; non N-fixing)}

There was no effect of soil moisture or $\mathrm{N}$ treatment, and no significant interactions among soil moisture, $\mathrm{N}$ treatment, and measurement date on $H$. multiflora percent growth (Table 1; Fig. 2a).

After accounting for measurement time, soil moisture did not influence $H$. multiflora chlorophyll fluorescence (Table 1; Fig. 2b). Marginal evidence suggested that $\mathrm{N}$ treatment influenced $H$. multiflora chlorophyll fluorescence (Table 1; Fig. 2b), with $\mathrm{N}$ additions increasing $\mathrm{F}_{\mathrm{v}} / \mathrm{F}_{\mathrm{m}}$ by $0.014 \mu \mathrm{mol}$ photons $\mathrm{m}^{-2} \mathrm{~s}^{-1}$ compared to control plants $\left(t_{1,21}=1.96\right.$, $p=0.064$; Table 2). There were no significant interactions among soil moisture, $\mathrm{N}$, and measurement date on H. multiflora chlorophyll fluorescence (Table 1).

After accounting for temperature, there was no effect of soil moisture or $\mathrm{N}$ treatment, and no interactions among soil moisture, $\mathrm{N}$, and measurement date on $H$. multiflora transpiration rate (Table 1; Fig. 2c).

After accounting for plant size, there was a significant interaction between soil moisture and $\mathrm{N}$ treatment on $H$. multiflora maximum floral display (Table 1; Fig. 2d). Floral display of control plants decreased by $7.0 \%$ per each $1 \%$ increase in soil moisture (95\% CI $0.9-14.0 \%$ decrease; Table 2), while the change in floral display across soil moisture for plants receiving $\mathrm{N}$ additions was not different from zero (95\% CI -5.0 to $7.2 \%$; Table 2 ). 

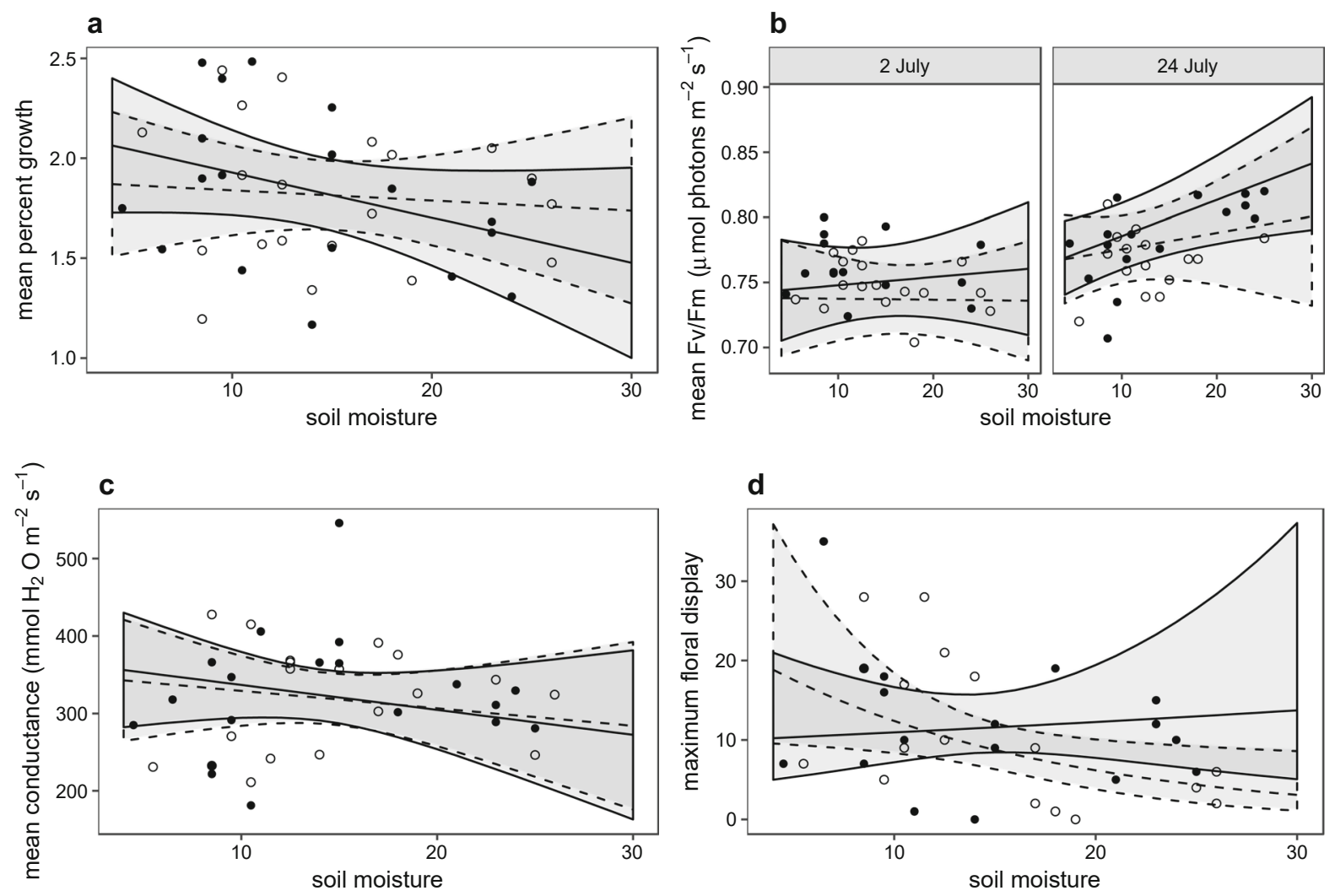

Fig. 2 Heliomeris multiflora. Least squares means and 95\% confidence bands for $\mathrm{N}$-addition (solid lines) and control plants (dashed lines) across soil moisture for a percent growth, b chlorophyll fluorescence, $\mathbf{c}$ transpiration rate, and $\mathbf{d}$ maximum

floral display. Observed data for $\mathrm{N}$-addition (filled circles) and control plants (open circles) are averaged across measurement dates in growth figures

Lathyrus leucanthus (shallow roots; N-fixing)

There was marginal evidence that soil moisture influenced L. leucanthus percent growth (Table 1; Fig. 3a). However, post hoc analyses suggested that the slope of growth across soil moisture was not different from zero (95\% CI - 0.9 to $5.6 \%$; Table 2). There was no effect of $\mathrm{N}$ treatment, and no significant interactions among soil moisture, $\mathrm{N}$ treatment, and measurement date on L. leucanthus percent growth (Table 1).

After accounting for measurement time, there was no effect of soil moisture on L. leucanthus chlorophyll fluorescence (Table 1; Fig. 3b). We observed a significant interaction between $\mathrm{N}$ treatment and measurement date on L. leucanthus chlorophyll fluorescence (Table 1; Fig. 3b). On 2 July, $\mathrm{N}$ additions increased $\mathrm{F}_{\mathrm{v}} / \mathrm{F}_{\mathrm{m}}$ by $0.036 \mu \mathrm{mol}$ photons $\mathrm{m}^{-2} \mathrm{~s}^{-1}$

compared to control plants $\left(t_{1,31}=4.01, p<0.001\right.$; Table 2), while $\mathrm{N}$ treatment did not affect chlorophyll fluorescence on 24 July ( $p=0.978$; Table 2 ). There was no significant interaction between soil moisture and $\mathrm{N}$ treatment or soil moisture and measurement date, and no significant three-way interaction of soil moisture, $\mathrm{N}$ treatment, and measurement date on $L$. leucanthus chlorophyll fluorescence (Table 1).

After accounting for temperature, there was no effect of soil moisture or $\mathrm{N}$ treatment, and no significant interactions among soil moisture, $\mathrm{N}$ treatment, and measurement date on L. leucanthus transpiration rate (Table 1; Fig. 3c).

After accounting for plant size, soil moisture influenced $L$. leucanthus maximum floral display (Table 1; Fig. 3d), with a $14.0 \%$ decrease in floral display per each $1 \%$ increase in soil moisture concentration (95\% CI 2.0-25.0\% decrease; Table 2). There 

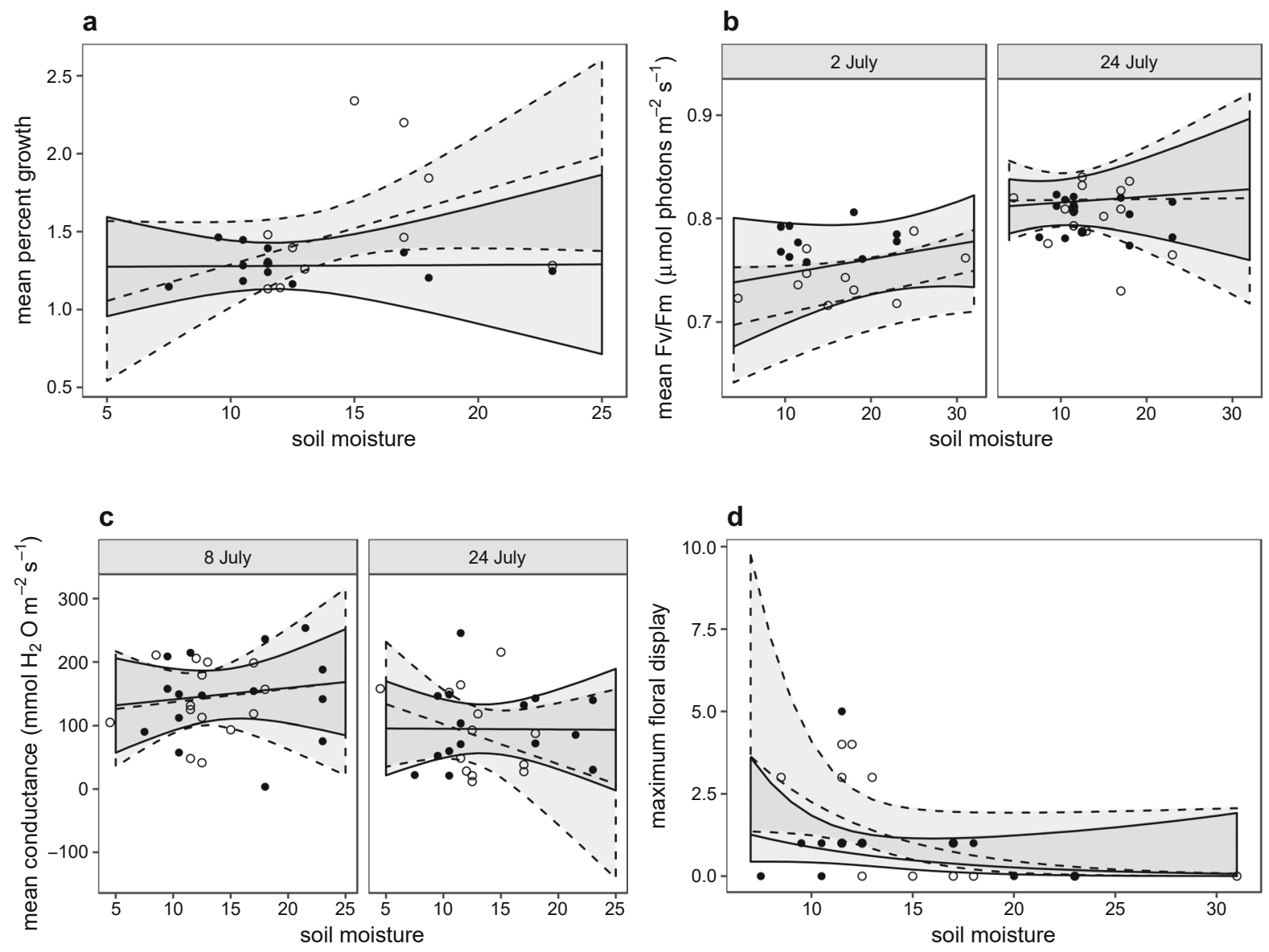

Fig. 3 Lathyrus leucanthus. Least squares means and 95\% confidence bands for $\mathrm{N}$-addition (solid lines) and control plants (dashed lines) across soil moisture for a percent growth, b chlorophyll fluorescence, $\mathbf{c}$ transpiration rate, and $\mathbf{d}$ maximum

floral display. Observed data for $\mathrm{N}$-addition (filled circles) and control plants (open circles) are averaged across measurement dates in growth figures

was no significant interaction between soil moisture and $\mathrm{N}$ treatment on $L$. leucanthus maximum floral display (Table 1).

\section{Lupinus spp. (deeper roots; $\mathrm{N}$-fixing)}

There was no effect of soil moisture or $\mathrm{N}$ treatment, and no significant interactions among soil moisture, $\mathrm{N}$ treatment, and measurement date on Lupinus spp. percent growth, chlorophyll fluorescence, or transpiration rate (Table 1; Fig. $4 \mathrm{a}-\mathrm{c}$ ).

After accounting for plant size, we observed marginal evidence that soil moisture influenced Lupinus spp. maximum floral display (Table 1; Fig. 4d), with floral display decreasing by $1.9 \%$ per each $1 \%$ increase in soil moisture concentration $(95 \% \mathrm{CI}$

1.9-3.6\% decrease; Table 2). There was no effect of $\mathrm{N}$ treatment and no significant interaction between soil moisture and $\mathrm{N}$ treatment on Lupinus spp. maximum floral display (Table 1; Fig. 4d).

\section{Discussion}

In this study, we investigated the growth and physiological responses of four subalpine forb species to $\mathrm{N}$ additions across a naturally-occurring soil moisture gradient in Colorado. Focal forb species differed in their rooting depths and $\mathrm{N}$-fixing abilities, two functional traits that we expected to influence their soil moisture and $\mathrm{N}$ limitations. Our objective was to assess how these functional traits mediated responses 

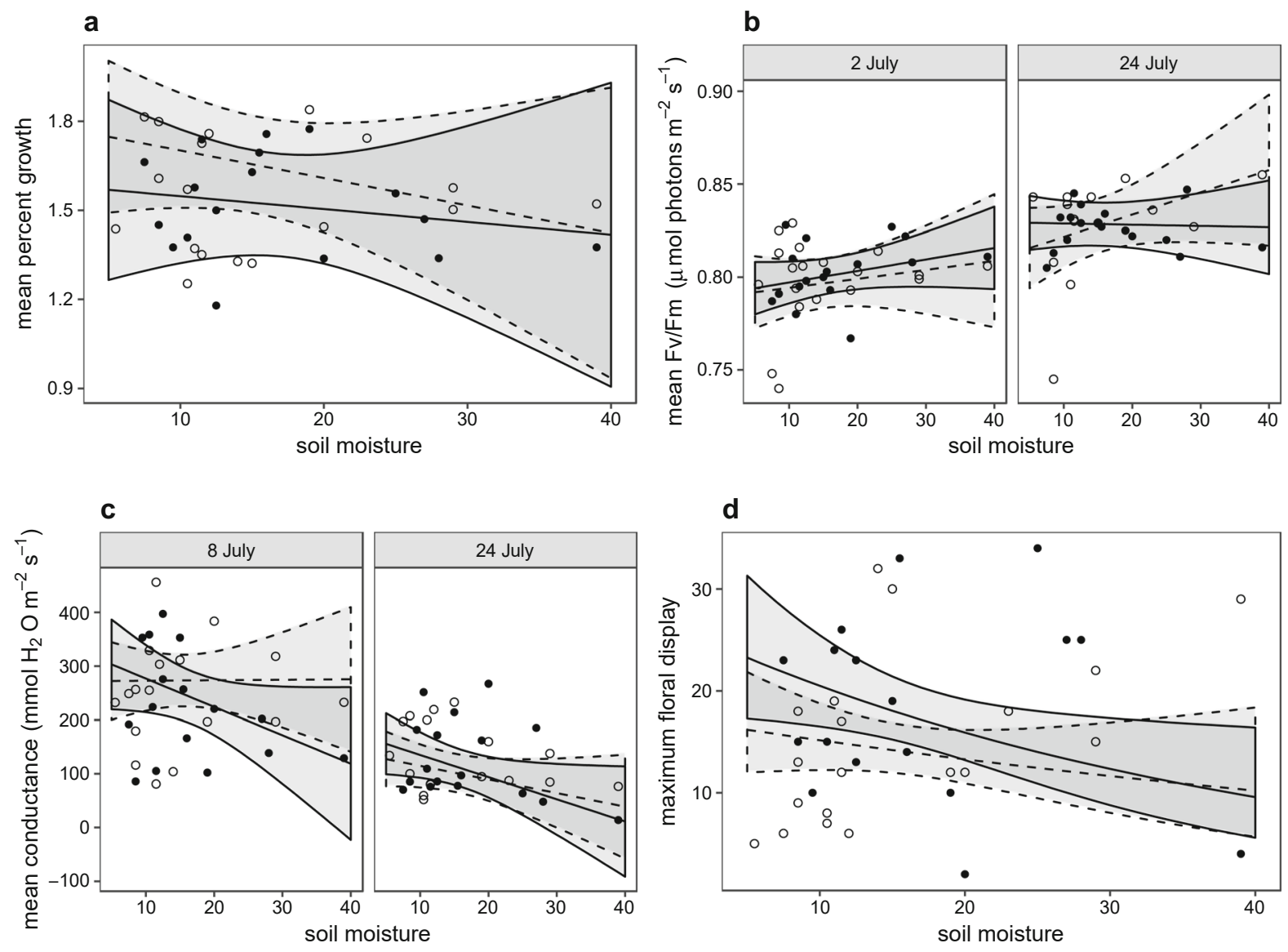

Fig. 4 Lupinus spp. Least squares means and 95\% confidence bands for $\mathrm{N}$-addition (solid lines) and control plants (dashed lines) across soil moisture for a percent growth, $\mathbf{b}$ chlorophyll fluorescence, $\mathbf{c}$ transpiration rate, and $\mathbf{d}$ maximum floral display.

to soil moisture and $\mathrm{N}$ availability. In addition, we used our results to speculate about how rooting depths and $\mathrm{N}$-fixing abilities may influence patterns of subalpine forb responses to anthropogenic changes in soil moisture and $\mathrm{N}$ concentrations. We expected positive responses to increased soil moisture by shallow-rooted species and we expected neutral or negative response to soil moisture by deeper-rooted species. Additionally, we expected positive responses to $\mathrm{N}$ additions by non-legume species and we expected neutral or negative response to $\mathrm{N}$ by legumes. The majority of our results were consistent with our expectations that plant responses to soil moisture and $\mathrm{N}$ availability would be influenced by resource limitations that reflect functional traits (De Valpine and Harte 2001). In some cases, soil moisture and N appeared to limit separate biological functions,

Observed data for $\mathrm{N}$-addition (filled circles) and control plants (open circles) are averaged across measurement dates in growth figures

highlighting the importance of assessing the effects of multiple resources on multiple plant functions. However, mixed support for our hypotheses suggests that environmental conditions and functional traits not evaluated in this study may also influence outcomes of resource competition for soil moisture and $\mathrm{N}$ in subalpine systems (Suding et al. 2005).

Growth responses to soil moisture were consistent with our expectations in three out of four species. Neither H. multiflora nor Lupinus spp. (both deeprooted) responded to soil moisture availability, while E. speciosus (shallow-rooted) grew larger as soil moisture increased. These findings support the common use of rooting depths to characterize drought susceptibility in plant species and highlight the role of soil moisture availability in structuring plant communities (Schenk and Jackson 2002). In the one 
exception, L. leucanthus (shallow-rooted) trended toward growing larger as soil moisture increased in control plants, but this trend was not statistically different from zero. The small stature of L. leucanthus may make this species a poor competitor for light, which could limit growth regardless of soil moisture availability (Tilman 1987). Although we did not observe a positive relationship between soil moisture and growth as predicted for the deeper-rooted species, we expected functional tradeoffs to reduce their competitive ability when soil moisture was abundant, resulting in reduced growth. The fact that we did not observe a negative relationship between growth and soil moisture in the deeper-rooted species suggests that mechanisms other than competition (e.g., facilitation) may influence plant community composition when resources are abundant (Callaway and Walker 1997). Taken together, the observed effects of soil moisture on growth suggest shallow-rooted species may be more vulnerable to increased drought stress compared to deeper-rooted species (Harte and Shaw 1995).

$\mathrm{N}$ additions did not affect growth in any species in this study. This was expected for legumes and suggests that legume growth is not limited by $\mathrm{N}$ (Suding et al. 2005; Skogen et al. 2011). For non-legumes, this was surprising given a preponderance of evidence suggesting that $\mathrm{N}$ commonly limits plant growth in temperature systems (e.g., Vitousek and Howarth 1991; Bobbink et al. 2010). Because our design combined field manipulations of $\mathrm{N}$ (i.e., short-term over one growing season) with a naturally-occurring soil moisture gradient (i.e., long-term, consistent plant exposure), responses to $\mathrm{N}$ may have been delayed (i.e., not observed over the duration of our study; Monaco et al. 2003; Burkle and Irwin 2009), while responses to soil moisture were evident. Alternatively, plants may have responded to $\mathrm{N}$ additions by allocating resources to below ground tissues (Magill et al. 1997), which were not measured in this study.

Photosynthetic capacity, evaluated as the ratio of variable chlorophyll fluorescence $\left(F_{v}\right)$ to maximal chlorophyll fluorescence $\left(\mathrm{F}_{\mathrm{m}}\right)$, responded to $\mathrm{N}$ as expected in three out of four species. $\mathrm{N}$ enhanced photosynthetic capacity in E. speciosus and H. multiflora (both non-legumes) and had no effect on Lupinus spp. (legume). In the one exception, $\mathrm{N}$ additions increased L. leucanthus (legume) photosynthetic capacity on one measurement date, which opposes our expectations of $\mathrm{N}$ limitation for $\mathrm{N}$-fixing species. Because drought stress can inhibit $\mathrm{N}$ fixation in root nodules of legume species (Serraj et al. 1999), shallow-rooted species like L. leucanthus may be more prone to $\mathrm{N}$ limitation compared to deeper-rooted legumes, possibly explaining the positive influence of $\mathrm{N}$ additions on the photosynthetic capacity of $L$. leucanthus. Indeed, Lupinus spp. (deep-rooted legume) did not respond positively to soil moisture or $\mathrm{N}$ additions. Additionally, competition for light may mediate photosynthetic output in short-statured species like L. leucanthus (Tilman 1987). Observed responses to $\mathrm{N}$ additions suggest that $\mathrm{N}$-fixing ability may be an indicator of $\mathrm{N}$ limitation on photosynthesis. If $\mathrm{N}$ limitation on photosynthetic capacity is an important indicator of which species will benefit from ongoing anthropogenic $\mathrm{N}$ deposition and which species may lose their competitive edge (Suding et al. 2005; Skogen et al. 2011), we expect N-fixing species to be more prone to declines. However, other factors, such as rooting depth and competition for light may also be important in some cases (Suding et al. 2005).

We observed mixed evidence that transpiration rates were influenced by soil moisture as expected based on rooting depths. As predicted, the two deeperrooted species maintained their transpiration rates across the soil moisture gradient. This suggests that deeper-rooted species may be less susceptible to drought (Schenk and Jackson 2002), potentially because they can access soil moisture at soil depths where moisture availability is greater and less variable. E. speciosus (shallow-rooted) transpiration rate increased as soil moisture decreased on one measurement date and L. leucanthus (shallow-rooted) transpiration rate did not respond to soil moisture on either date. The transpiration responses by E. speciosus and L. leucanthus suggest that the mechanisms that regulate transpiration rates may involve complex responses to environmental conditions, such as changes in plant-water relations that occur in response to temperature over the course of the day (Johnson et al. 2009). Indeed, temperature was the strongest predictor of transpiration rates in three out of four species in this study. The properties of the leaf boundary layer, which can depend on temperature, humidity, and $\mathrm{CO}_{2}$ concentration at the leaf surface, may also regulate stomatal aperture and subsequent water loss through stomata (Collatz et al. 1991). Thus, 
we interpret the transpiration responses by the forbs in this study with caution and acknowledge that soil moisture limitations and soil moisture availability may interact with other mechanisms to influence transpiration rates in plants.

We observed mixed evidence that maximum floral displays were influenced by soil moisture and $\mathrm{N}$ as expected based on rooting depths and $\mathrm{N}$-fixing abilities. Maximum floral displays decreased as soil moisture increased for $H$. multiflora control plants and E. speciosus $\mathrm{N}$-addition plants, as well as Lupinus spp. and L. leucanthus across $\mathrm{N}$-addition and control plants. Floral display responses observed for $H$. multiflora and Lupinus spp. (both deeper-rooted species), suggest that tradeoffs in rooting depths may reduce the competitive ability of deep-rooted species when soil moisture is abundant and other soil nutrients may be more limiting (Ho et al. 2005). Indeed, $H$. multiflora floral display (deeper-rooted, non-legume) was not affected by soil moisture when $\mathrm{N}$ was added, suggesting that $H$. multiflora floral display may be limited by $\mathrm{N}$ when soil moisture is abundant. Unexpectedly, E. speciosus (shallow-rooted, non-legume) floral display decreased as soil moisture increased in $\mathrm{N}$-addition plants, while control plants were not affected by soil moisture. One possible explanation for this result is that E. speciosus may have allocated moisture and $\mathrm{N}$ resources to growth at the expense of floral display (Primack \& Hall 1990). This is reasonable given soil moisture and $\mathrm{N}$ positively influenced $E$. speciosus growth and photosynthetic capacity, respectively. In addition, the effect of $\mathrm{N}$ on E. speciosus floral display may have been delayed beyond our study period (Monaco et al. 2003; Burkle and Irwin 2009). A negative response to soil moisture by L. leucanthus (shallow-rooted), along with no evidence that $L$. leucanthus was allocating soil moisture resources to growth, suggests that soil moisture may not limit $L$. leucanthus floral display as reported for other forbs (Campbell and Halama 1993; Burkle and Irwin 2009; but see Galen 1999). Alternatively, the unanticipated responses by E. speciosus and L. leucanthus floral displays may indicate that their floral displays are limited by a soil nutrient that was not measured in the study, such as phosphorous (Burkle \& Irwin 2009). N treatment did not affect floral displays in either legume species, suggesting that floral displays of $\mathrm{N}$-fixing species are not limited by $\mathrm{N}$ (Suding et al. 2005; Burkle and Irwin 2010; Skogen et al. 2011).
Despite previously reported evidence of co-limitation by $\mathrm{N}$ and soil moisture (Harpole et al. 2007; Reich et al. 2014; Vourlitis et al. 2017) in grasslands, we did not observe positive soil moisture $\times \mathrm{N}$ interactions in this study. Since we did not measure background levels of $\mathrm{N}$ or other soil nutrients at our study site, it is possible that co-limitation by soil nutrients other than $\mathrm{N}$ and soil moisture (Harpole et al. 2011), or covariance between $\mathrm{N}$ and soil moisture (Burke et al. 1997) explains why we did not observe positive soil moisture $\times \mathrm{N}$ interactions. However, independent main effects of soil moisture and $\mathrm{N}$ treatment on $E$. speciosus growth and photosynthetic capacity suggest that $\mathrm{N}$ and soil moisture both influence key biological processes in this species, which constitutes evidence of co-limitation by $\mathrm{N}$ and soil moisture in E. speciosus.

Here, we evaluated the influence of functional traits on subalpine forb responses to soil moisture and $\mathrm{N}$ availability. The majority of responses were consistent with expected $\mathrm{N}$ and soil moisture limitations reflecting the rooting depths and $\mathrm{N}$-fixing abilities of the forbs in this study. Based on this evaluation, we speculate that shallow-rooted forbs and forbs with $\mathrm{N}$-fixing abilities may be more prone to declines under anthropogenic increases in drought severity and $\mathrm{N}$ availability. However, mixed support for our hypotheses highlights that environmental conditions and functional traits not evaluated by this study may be important in determining subalpine forb responses to soil moisture and $\mathrm{N}$ availability. We caution that concentrated applications of $\mathrm{N}$ over one season (i.e., a nutrient pulse) may not necessarily represent plant responses to less concentrated, long-term inputs occurring via anthropogenic deposition (i.e., a nutrient press; Murphy et al. 2012). We also acknowledge that plant responses to $\mathrm{N}$ treatments may not be evident in one season and that interpreting responses to $\mathrm{N}$ treatments conducted over a single season may not reflect plant responses to long-term $\mathrm{N}$ enrichment (Inouye and Tilman 1995). We urge future studies to extend similar field manipulations across naturallyoccurring resource gradients over multiple years to further discern short- and long-term patterns of plant responses to increasing $\mathrm{N}$ availability (Arft et al. 1999; Dunne et al. 2004). To help disentangle the influence of functional traits on competition for limiting resources, we stress the need for future research to assess the effects of concurrent changes in multiple 
resources and to measure multiple response variables that reflect several plant functions.

Acknowledgements We thank the Rocky Mountain Biological Lab for logistical considerations and two anonymous reviewers for providing insightful comments on the manuscript. The Rocky Mountain Biological Lab Advanced Undergraduate Research Program, Western State Colorado University Thornton Research Grants Program, and Montana State University provided funding to Anthony Slominski for this project.

\section{References}

Arft AM, Walker MD, Gurevitch J, Alatalo JM, Bret-Harte MS, Dale M, Diemer M, Gugerli F, Henry GHR, Jones MH, Hollister RD, Jonsdottir IS, Laine K, Levesque E, Marion GM, Molau U, Molgaard P, Nordenhall U, Raszhivin V, Robinson CH, Starr G, Stenstrom A, Stenstrom M, Totland O, Turner PL, Walker LJ, Webber PJ, Welker JM, Wookey PA (1999) Responses of tundra plants to experimental warming: meta-analysis of the international tundra experiment. Ecol Monogr 69:491-511

Baker NR (2008) Chlorophyll fluorescence: a probe of photosynthesis in vivo. Annu Rev Plant Biol 59:89-113

Ball MC, Butterworth JA, Roden JS, Christian R, Egerton JJG (1995) Applications of chlorophyll fluorescence to forest ecology. Aust J Plant Physiol 22:311-319

Bobbink R, Hicks K, Galloway J, Spranger T, Alkemade R, Ashmore M, Bustamante M, Cinderby S, Davidson E, Dentener F, Emmett B, Erisman JW, Fenn M, Gilliam F, Nordin A, Pardo L, De Vries W (2010) Global assessment of nitrogen deposition effects on terrestrial plant diversity: a synthesis. Ecol Appl 20:30-59

Bond BJ, Kavanagh KL (1999) Stomatal behavior of four woody species in relation to leaf-specific hydraulic conductance and threshold water potential. Tree Physiol 19:503-510

Bowman WD, Gartner JR, Holland K, Wiedermann M (2006) Nitrogen critical loads for alpine vegetation and terrestrial ecosystem response: are we there yet? Ecol Appl 16:1183-1193

Burke IC, Lauenroth WK, Parton WJ (1997) Regional and temporal variation in net primary production and nitrogen mineralization in grasslands. Ecology 78:1330-1340

Burkle LA, Irwin RE (2009) The effects of nutrient addition on floral characters and pollination in two subalpine plants, Ipomopsis aggregata and Linum lewisii. Plant Ecol 203:83-98

Burkle LA, Irwin RE (2010) Beyond biomass: measuring the effects of community-level nitrogen enrichment on floral traits, pollinator visitation and plant reproduction. J Ecol 98:705-717

Callaway RM, Walker LR (1997) Competition and facilitation: a synthetic approach to interactions in plant communities. Ecology 78:1958-1965
Campbell DR, Halama KJ (1993) Resource and pollen limitations to lifetime seed production in a natural plant-population. Ecology 74:1043-1051

Chapin FS III, Zavaleta ES, Eviner VT, Naylor RL, Vitousek PM, Reynolds HL, Hooper DU, Lavorel S, Sala OE, Hobbie SE, Mack MC, Diaz S (2000) Consequences of changing biodiversity. Nature (London) 405:234-242

Chaves MM, Pereira JS, Maroco J, Rodrigues ML, Ricardo CPP, Osorio ML, Carvalho I, Faria T, Pinheiro C (2002) How plants cope with water stress in the field Photosynthesis and growth. Ann Bot 89:907-916

Clark CM, Tilman D (2008) Loss of plant species after chronic low-level nitrogen deposition to prairie grasslands. Nature 451:712-715

Cleland EE (2010) Harpole WS (2010) Nitrogen enrichment and plant communities. Year Ecol Conserv Biol 1195:46-61

Collatz GJ, Ball JT, Grivet C, Berry JA (1991) Physiological and environmental-regulation of stomatal conductance, photosynthesis and transpiration-a model that includes a laminar boundary-layer. Agric For Meteorol 54:107-136

Cronquist A, Holmgren NH, Holmgren PK, Reveal JL, Barneby RC, Garden New York Botanical (1972) Intermountain flora; vascular plants of the Intermountain West. Published for the New York Botanical Garden by Hafner Pub Co, New York

Dai A (2013) Increasing drought under global warming in observations and models. Nature Climate Change 3:52-58

De Valpine P, Harte J (2001) Plant responses to experimental warming in a montane meadow. Ecology (Durham) 82:637-648

Dickson TL, Mittelbach GG, Reynolds HL, Gross KL (2014) Height and clonality traits determine plant community responses to fertilization. Ecology 95:2443-2452

Dukes JS, Chiariello NR, Cleland EE, Moore LA, Shaw MR, Thayer S, Tobeck T, Mooney HA, Field CB (2005) Responses of grassland production to single and multiple global environmental changes. PLoS Biol 3:1829-1837

Dunne JA, Saleska SR, Fischer ML, Harte J (2004) Integrating experimental and gradient methods in ecological climate change research. Ecology 85:904-916

Dybzinski R, Tilman D (2007) Resource use patterns predict long-term outcomes of plant competition for nutrients and light. Am Nat 170:305-318

Farquhar GD, Sharkey TD (1982) Stomatal conductance and photosynthesis. Annu Rev Plant Physiol Plant Mol Biol 33:317-345

Fitter AH, Stickland TR, Harvey ML, Wilson GW (1991) Architectural analysis of plant-root systems.1. Architectural correlates of exploitation efficiency. New Phytol 118:375-382

Foster BL, Gross KL (1998) Species richness in a successional grassland: effects of nitrogen enrichment and plant litter. Ecology 79:2593-2602

Fowler D, Coyle M, Skiba U, Sutton MA, Cape JN, Reis S, Sheppard LJ, Jenkins A, Grizzetti B, Galloway JN, Vitousek P, Leach A, Bouwman AF, Butterbach-Bahl K, Dentener F, Stevenson D, Amann M, Voss M (2013) The global nitrogen cycle in the twenty-first century. Philos Trans Royal Soc B Biol Sci 368:13 
Galen C (1999) Why do flowers vary? The functional ecology of variation in flower size and form within natural plant populations. Bioscience 49:631-640

Gardner WR (1960) Dynamic aspects of water availability to plants. Soil Sci 89:63-73

Genty B, Briantais JM, Baker NR (1989) The relationship between the quantum yield of photosynthetic electrontransport and quenching of chlorophyll fluorescence. Biochem Biophys Acta 990:87-92

Granier A, Loustau D, Breda N (2000) A generic model of forest canopy conductance dependent on climate, soil water availability and leaf area index. Ann For Sci 57:755-765

Grime JP (2006) Trait convergence and trait divergence in herbaceous plant communities: mechanisms and consequences. J Veg Sci 17:255-260

Harpole WS, Potts DL, Suding KN (2007) Ecosystem responses to water and nitrogen amendment in a California grassland. Glob Change Biol 13:2341-2348

Harpole WS, Ngai JT, Cleland EE, Seabloom EW, Borer ET, Bracken MES, Elser JJ, Gruner DS, Hillebrand H, Shurin JB, Smith JE (2011) Nutrient co-limitation of primary producer communities. Ecol Lett 14:852-862

Harte J, Shaw R (1995) Shifting dominance within a montane vegetation community-results of a climate-warming experiment. Science 267:876-880

Harte J, Torn MS, Chang FR, Feifarek B, Kinzig AP, Shaw R, Shen K (1995) Global warming and soil microclimateresults from a meadow-warming experiment. Ecol Appl $5: 132-150$

Ho MD, Rosas JC, Brown KM, Lynch JP (2005) Root architectural tradeoffs for water and phosphorus acquisition. Funct Plant Biol 32:737-748

Hooper DU, Chapin FS, Ewel JJ, Hector A, Inchausti P (2005) Effects of biodiversity on ecosystem functioning: a consensus of current knowledge. Ecol Monogr 75:3-35

Hoover DL, Duniway MC, Belnap J (2017) Testing the apparent resistance of three dominant plants to chronic drought on the Colorado Plateau. J Ecol 105:152-162

Inouye DW (2008) Effects of climate change on phenology, frost damage, and floral abundance of montane wildflowers. Ecology 89:353-362

Inouye RS, Tilman D (1995) Convergence and divergence of old-field vegetation after $11 \mathrm{yr}$ of nitrogen addition. Ecology 76:1872-1887

Inouye DW, Morales MA, Dodge GJ (2002) Variation in timing and abundance of flowering by Delphinium barbeyi Huth (Ranunculaceae): the roles of snowpack, frost, and La Nina, in the context of climate change. Oecologia 130:543-550

IPCC (2014): Climate Change 2014: Synthesis Report Contribution of Working Groups I, II, and III to the Fifth Assessment Report of the Intergovernmental Panel on Climate Change [Core Writing Team, RK Pachauri and LA Meyer (eds)] IPCC, Geneva, Switzerland, 151

Jacobs DE, Salifu KF, Davis AS (2009) Drought susceptibility and recovery of transplanted Quercus rubra seedlings in relation to root system morphology. Ann For Sci 66:1-12

Johnson DM, Woodruff DR, McCulloh KA, Meinzer FC (2009) Leaf hydraulic conductance, measured in situ, declines and recovers daily: leaf hydraulics, water potential and stomatal conductance in four temperate and three tropical tree species. Tree Physiol 29:879-887

Knapp AK, Fay PA, Blair JM, Collins SL, Smith MD, Carlisle JD, Harper CW, Danner BT, Lett MS, McCarron JK (2002) Rainfall variability, carbon cycling, and plant species diversity in a mesic grassland. Science 298:2202-2205

Lavorel S, Garnier E (2002) Predicting changes in community composition and ecosystem functioning from plant traits: revisiting the Holy Grail. Funct Ecol 16:545-556

Lenth RV, HervÃ M (C (2015) lsmeans: Least-Squares Means R package version 216. http://www.CRANR-projectorg/ package $=$ lsmeans

Leverenz JW, Öquist G (1987) Quantum yields of photosynthesis at temperatures between -2-degrees $c$ and 35-degrees $\mathrm{c}$ in a cold-tolerant c-3 plant (pinus-sylvestris) during the course of one year. Plant, Cell Environ 10:287-295

Lynch J (1995) Root architecture and plant productivity. Plant Physiol 109:7-13

Magill AH, Aber JD, Hendricks JJ, Bowden RD, Melillo JM, Steudler PA (1997) Biogeochemical response of forest ecosystems to simulated chronic nitrogen deposition. Ecol Appl 7:402-415

Monaco TA, Johnson DA, Norton JM, Jones TA, Connors KJ, Norton JB, Redinbaugh MB (2003) Contrasting responses of intermountain west grasses to soil nitrogen. J Range Manag 56:282-290

Mote PW (2006) Climate-Driven Variability and Trends in Mountain Snowpack in Western North America. J Clim 19:6209-6220

Murphy SM, Wimp GM, Lewis D, Denno RF (2012) Nutrient presses and pulses differentially impact plants, herbivores, detritivores and their natural enemies. PLoS ONE 7:e43929

NOAA; https://www.ncdc.noaa.gov/

Obidiegwu JE, Bryan GJ, Jones HG, Prashar A (2015) Coping with drought: stress and adaptive responses in potato and perspectives for improvement. Front Plant Sci 6:542

Parmesan C, Hanley ME (2015) Plants and climate change: complexities and surprises. Ann Bot 116:849-864

Pecl GT, Araujo MB, Bell JD, Blanchard J, Bonebrake TC, Chen IC, Clark TD, Colwell RK, Danielsen F, Evengard B, Falconi L, Ferrier S, Frusher S, Garcia RA, Griffis RB, Hobday AJ, Janion-Scheepers C, Jarzyna MA, Jennings S, Lenoir J, Linnetved HI, Martin VY, McCormack PC, McDonald J, Mitchell NJ, Mustonen T, Pandolfi JM, Pettorelli N, Popova E, Robinson SA, Scheffers BR, Shaw JD, Sorte CJB, Strugnell JM, Sunday JM, Tuanmu M-N, Verges A, Villanueva C, Wernberg T, Wapstra E, Williams SE (2017) Biodiversity redistribution under climate change: impacts on ecosystems and human well-being. Science (New York, NY) 355

Pinheiro, Jose, Bates D, DebRoy S, Sarkar, D, the R Development Core Team (2013) nlme: Linear and Nonlinear Mixed Effects Models R package version 31-111

Primack RB, Hall P (1990) Costs of reproduction in the pink ladys-slipper orchid-a 4-year experimental-study. Am Nat 136:638-656

R Core Team (2017) R: A language and environment for statistical computing. R Foundation for Statistical Computing,Vienna, Austria. http://www.R-projectorg/ 
Reich PB, Hobbie SE, Lee TD (2014) Plant growth enhancement by elevated $\mathrm{CO} 2$ eliminated by joint water and nitrogen limitation. Nat Geosci 7:920-924

Sack L, Holbrook NM (2006) Leaf hydraulics. Annu Rev Plant Biol 57:361-381

Sala OE, Chapin FS, Armesto JJ, Berlow E, Bloomfield J, Dirzo R, Huber-Sanwald E, Huenneke LF, Jackson RB, Kinzig A, Leemans R, Lodge DM, Mooney HA, Oesterheld M, Poff NL, Sykes MT, Walker BH, Walker M, Wall DH (2000) Biodiversity - Global biodiversity scenarios for the year 2100. Science 287:1770-1774

Schenk HJ, Jackson RB (2002) The global biogeography of roots. Ecol Monogr 72:311-328

Serraj R, Sinclair TR, Purcell LC (1999) Symbiotic N-2 fixation response to drought. J Exp Bot 50:143-155

Shantz AA, Lemoine NP, Burkepile DE (2016) Nutrient loading alters the performance of key nutrient exchange mutualisms. Ecol Lett 19:20-28

Skogen KA, Holsinger KE, Cardon ZG (2011) Nitrogen deposition, competition and the decline of a regionally threatened legume, Desmodium cuspidatum. Oecologia 165:261-269

Smith MD, La Pierre KJ, Collins SL, Knapp AK, Gross KL, Barrett JE, Frey SD, Gough L, Miller RJ, Morris JT, Rustad LE, Yarie J (2015) Global environmental change and the nature of aboveground net primary productivity responses: insights from long-term experiments. Oecologia 177:935-947

Stevens CJ, Dise NB, Mountford JO, Gowing DJ (2004) Impact of nitrogen deposition on the species richness of grasslands. Science 303:1876-1879

Stewart IT (2009) Changes in snowpack and snowmelt runoff for key mountain regions. Hydrol Process 23:78-94

Suding KN, Collins SL, Gough L, Clark C, Cleland EE, Gross KL, Milchunas DG, Pennings S (2005) Functional- and abundance-based mechanisms explain diversity loss due to N fertilization. Proc Natl Acad Sci USA 102:4387-4392

Tilman D (1987) Secondary succession and the pattern of plant dominance along experimental nitrogen gradients. Ecol Monogr 57:189-214
Tuzet A, Perrier A, Leuning R (2003) A coupled model of stomatal conductance, photosynthesis and transpiration. Plant, Cell Environ 26:1097-1116

USDA Plant Database, https://www.plants.usda.gov/

van der Tol C, Berry JA, Campbell PKE, Rascher U (2014) Models of fluorescence and photosynthesis for interpreting measurements of solar-induced chlorophyll fluorescence. J Geophys Res Biogeosci 119:2312-2327

Venables WN, Ripley BD (2002) Modern applied statistics with S, 4th Edn. Springer, New York

Vitousek PM, Howarth RW (1991) Nitrogen limitation on land and in the sea - how can it occur? Biogeochemistry 13:87-115

Vitousek PM, Aber JD, Howarth RW, Likens GE, Matson PA, Schindler DW, Schlesinger WH, Tilman D (1997) Human alteration of the global nitrogen cycle: sources and consequences. Ecol Appl 7:737-750

Vourlitis GL (2017) Chronic N enrichment and drought alter plant cover and community composition in a Mediterranean-type semi-arid shrubland. Oecologia 184:267-277

Wei X, Reich PB, Hobbie SE, Kazanski CE (2017) Disentangling species and functional group richness effects on soil $\mathrm{N}$ cycling in a grassland ecosystem. Glob Change Biol $00: 1-11$

Wickham H (2009) Ggplot2: elegant graphics for data analysis. Springer, New York

Williams MW, Baron JS, Caine N, Sommerfeld R, Sanford R (1996) Nitrogen saturation in the Rocky Mountains. Environ Sci Technol 30:640-646

Yue K, Fornara DA, Yang W, Peng Y, Peng C, Liu Z, Wu F (2017) Influence of multiple global change drivers on terrestrial carbon storage: additive effects are common. Ecol Lett 20:663-672

Zavaleta ES, Shaw MR, Chiariello NR, Thomas BD, Cleland EE, Field CB, Mooney HA (2003) Grassland responses to three years of elevated temperature, $\mathrm{CO}(2)$, precipitation, and $\mathrm{N}$ deposition. Ecol Monogr 73:585-604

Zuur AF, Ieno EN, Walker NJ, Saveliev AA, Smith GM (2009) Mixed effects models and extensions in ecology with R. Springer, New York 